\title{
A ZOO OF GROWTH FUNCTIONS OF MAPPING CLASS SETS
}

\author{
FEDOR MANIN
}

\begin{abstract}
Suppose $X$ and $Y$ are finite complexes, with $Y$ simply connected. Gromov conjectured that the number of mapping classes in $[X, Y]$ which can be realized by $L$ Lipschitz maps grows asymptotically as $L^{\alpha}$, where $\alpha$ is an integer determined by the rational homotopy type of $Y$ and the rational cohomology of $X$. This conjecture was disproved in a recent paper of the author and Weinberger; we gave an example where the "predicted" growth is $L^{8}$ but the true growth is $L^{8} \log L$. Here we show, via a different mechanism, that the universe of possible such growth functions is quite large. In particular, for every rational number $r \geq 4$, there is a pair $X, Y$ for which the growth of $[X, Y]$ is essentially $L^{r}$.
\end{abstract}

\section{INTRODUCTION}

Let $X$ and $Y$ be compact piecewise Riemannian spaces, for example Riemannian manifolds with boundary or finite simplicial complexes with a simplexwise linear metric. The growth of the mapping class set $[X, Y]$ is the function $g_{[X, Y]}: \mathbb{R}^{+} \rightarrow \mathbb{N}$ given by

$$
g_{[X, Y]}(L)=\#\{\alpha \in[X, Y] \text { which have a representative with Lipschitz constant } \leq L\} .
$$

Asymptotically, this is a topological and even homotopy-theoretic notion; this is because any compact, homotopy equivalent $X$ and $X^{\prime}$ of this form are in fact Lipschitz homotopy equivalent.

One well-studied class of examples is the growth of a finitely presented group $\Gamma$, which can be thought of as the growth of the set of (based) homotopy classes of maps $S^{1} \rightarrow Y$, for any complex $Y$ with fundamental group $\Gamma$. In that setting, Gromov's famous polynomial growth theorem Gro81] states that growth is either asymptotic to a polynomial (for virtually nilpotent groups) or superpolynomial (for all other groups). In this paper, we instead concern ourselves with simply connected $Y$ and general $X$, where the picture turns out to be very different.

This notion of growth was first introduced by Gromov in Gro78, where he noted several facts about it. First, he showed that the growth of $\left[S^{n}, S^{n}\right]$ is $\Theta\left(L^{n}\right)$. The upper bound is essentially homological: the domain of an $L$-Lipschitz map can fit at most $O\left(L^{n}\right)$ preimages of a regular point, counted with sign. (We later give the simplest rigorous formulation of this argument, which uses differential forms.) Similar upper bounds can be found for the pullback of any cohomology class.

Next, Gromov showed that the growth of $\left[S^{4 n-1}, S^{2 n}\right]$ is $\Theta\left(L^{4 n}\right)$, rather than the $\Theta\left(L^{4 n-1}\right)$ one might expect by generalizing from the previous estimate. The upper bound here generalizes to show that $g_{\left[S^{n}, Y\right]}$ is bounded by a polynomial when $Y$ is simply connected. (We are nowhere close to showing whether this bound is sharp.)

In Gro98, Ch. 7, p. 358]1, Gromov gave some further conjectures about $g_{[X, Y]}$ in the case where $Y$ is simply-connected; notably, he conjectured that it is always asymptotic to $d^{\alpha}$, where $\alpha$ is an integer determined by calculations in rational homotopy theory.

\footnotetext{
${ }^{1}$ The same range of ideas is discussed in Gromov's conference paper Gro99.
} 
The heuristic that leads to this conjecture is as follows. By obstruction theory, a map $f: X \rightarrow Y$ is determined, non-uniquely and up to some finite indeterminacy, by a finite number of $(\mathbb{Q}$-valued) rational homotopy invariants. Gromov sketches an argument that these invariants are always bounded by a polynomial in the Lipschitz constant, and therefore (informally speaking) all homotopy classes of $L$-Lipschitz maps live in a region of polynomial volume in the set of rational homotopy classes. If one makes a pair of very strong assumptions, informally stated as follows:

(S1) integral homotopy classes are sprinkled evenly through this region

(S2) and all integral classes in the region are realizable via $L$-Lipschitz maps,

then the conjecture holds.

In MW18, the author and Weinberger showed that $g_{[X, Y]}$ is bounded by a polynomial whenever $Y$ is simply connected. However, we disproved the stronger conjecture by showing that

$$
g_{\left[\left(S^{3} \times S^{4}\right) \# 2, S^{4}\right]}(L)=\Theta\left(L^{8} \log L\right) .
$$

This construction relied on violating assumption (S1): for this example, the "density" of integral homotopy classes in the rational ones grows without bound as rational invariants increase.

In this paper we show that assumption (S2) - that all homotopy classes whose rational homotopy invariants satisfy the obvious bounds are realizable with Lipschitz constant $O(L)$ is also false in general. This lets us produce a much larger variety of growth functions of mapping class sets. In fact, we prove the following:

Theorem A. For every rational number $r>4$, there is a pair of simply connected spaces $X$ and $Y$ such that $\lim _{L \rightarrow \infty} \log _{L}\left(g_{[X, Y]}(L)\right)=r$.

In other words, for every sufficiently large rational $r$ we have a growth function which is more like $L^{r}$ than $L^{r^{\prime}}$ for any other $r^{\prime} \in \mathbb{Q}$. A plausible conjecture about Lipschitz nullhomotopies implies that this growth function is in fact $\Theta\left(L^{r}\right)$, but for now we cannot exclude the possibility that it is slightly slower. In addition, we give another example whose growth function includes a logarithm.

Techniques. All of these examples are constructed in essentially the same way. The space $X$ consists of a wedge of two spheres $S^{a} \vee S^{b}$ of different dimensions, together with a single third cell of some high dimension $n$; the space $Y$ is obtained from $X$ by gluing on additional $(n+1)$ cells, killing $\pi_{n}(X)$. A cellular map $X \rightarrow Y$ is determined (up to a bounded indeterminacy) by its degrees on the $a$ - and $b$-cells; this in turn determines the degree on the higher-dimensional cell, putting an extra constraint on what the two invariants can be for an $L$-Lipschitz map.

Another way of seeing this is via Lipschitz nullhomotopies. Any map $f: S^{a} \vee S^{b} \rightarrow Y$ (which is determined by two obstructions in $\mathbb{Z}$ ) extends uniquely up to homotopy to $X$, via a nullhomotopy of $f \circ \partial$, where $\partial$ is the attaching map of the top cell. However, this extension may be forced to have a much larger Lipschitz constant. In other words, while there is no absolute obstruction to performing the extension, there is a quantitative obstruction in the form of a relatively long finite bar in the persistence homotopy of $\pi_{n-1}(Y)$.

This phenomenon is closely related to another in quantitative homotopy theory: the distortion of homotopy group elements. The distortion function of an element $\alpha \in \pi_{n}(Y)$ is

$$
\delta_{\alpha}(k)=\inf \left\{\operatorname{Lip} f \mid f: S^{n} \rightarrow Y,[f]=k \alpha\right\} .
$$


In the given examples, the attaching map $S^{n-1} \stackrel{\partial}{\rightarrow} S^{3} \vee S^{4}$ of the top cell has a slowgrowing distortion function; in other words, for large $N \in \mathbb{Z}$, there is a relatively small map $S^{n-1} \rightarrow S^{3} \vee S^{4}$ whose homotopy class is $N[\partial]$. However, in order to nullhomotope this map in $Y$, one still needs a homotopy which has relative degree $N$ over the $n$-cell; this means that the Lipschitz constant of the homotopy is forced to be at least $\sim N^{1 / n}$.

Outline of the paper. Section 2 presents various background information needed for the main constructions. We invite the reader to skip it and refer back to it as needed. The zoo advertised in the title is presented in Section 3. Section 4 discusses some situations in which the assumption (S2) holds and therefore Gromov's prediction is closer to correct.

\section{TECHNicAl BACKGROUND}

2.1. Cellular maps. To enumerate homotopy classes between $\mathrm{CW}$ complexes, it is enough to consider cellular maps. The same turns out to be the case if we want to enumerate those with an $L$-Lipschitz representative (up to a constant depending on the metrics on $X$ and $Y$ ).

Lemma 2.1. Let $X$ and $Y$ be piecewise Riemannian finite $C W$ complexes with Lipschitz attaching maps. Then any L-Lipschitz map $X \rightarrow Y$ can be deformed to a cellular one whose Lipschitz constant is $C(X, Y)(L+1)$.

Thus if every cellular representative of a homotopy class is $\geq L$-Lipschitz, then every representative is $\geq(L-1) / C$-Lipschitz.

Proof. We can convert $X$ and $Y$ into homotopy equivalent simplicial complexes $X^{\prime}$ and $Y^{\prime}$ skeleton-by-skeleton: at each stage, the attaching maps of cells in the next dimension are homotopic to simplicial maps on some triangulation of the boundary of the cell. In particular, if we put the standard metric on each simplex of $X^{\prime}$ and $Y^{\prime}$, we get Lipschitz homotopy equivalences in both directions which we can also ensure are cellular.

Now, given an $L$-Lipschitz map $f: X \rightarrow Y$, we can pre- and postcompose to get a $C_{1} L$-Lipschitz map $X^{\prime} \rightarrow Y^{\prime}$. By the Lipschitz simplicial approximation theorem given in CDMW18, this map is homotopy equivalent to a simplicial map on a suitable subdivision of $X^{\prime}$ which is $C_{2}\left(C_{1} L+1\right)$-Lipschitz. Finally we pre- and postcompose to get a cellular map $X \rightarrow Y$.

So if we want an upper bound on the growth of $[X, Y]$, it is enough to obtain an upper bound on the number of homotopy classes of $L$-Lipschitz cellular maps in terms of $L$. One way of obtaining such upper bounds is via degree considerations:

Lemma 2.2. Suppose that $f: X \rightarrow Y$ is a cellular L-Lipschitz map. Then for any $n$-cell $\sigma$ of $X$ and $n$-cell $\tau$ of $Y$, the induced map

$$
(\sigma, \partial \sigma) \rightarrow\left(Y^{(n)}, Y^{(n)} \backslash \operatorname{int}(\tau)\right)
$$

has relative degree $O\left(L^{n}\right)$.

Proof. Since $f$ can be approximated by an $(L+\varepsilon)$-Lipschitz smooth map, we can assume that it is smooth. Let $\omega$ be an $n$-form supported on the interior of $\tau$ with $\int_{\tau} \omega=1$ and $\|\omega\|_{\infty} \leq C$. (Here the $\infty$-norm of a form is the supremum over its values on all frames of unit vectors.) Then

$$
\left|\int_{\sigma} f^{*} \omega\right| \leq \operatorname{vol} \sigma\left\|f^{*} \omega\right\|_{\infty} \leq C \operatorname{vol} \sigma|\operatorname{Lip} f|^{n}
$$

But this integral is the relative degree we are looking for. 
2.2. Nullhomotopy and extension. Suppose that we are constructing a cellular map $X \rightarrow$ $Y$ skeleton-by-skeleton. We would like to extend a map $f_{n-1}: X^{(n-1)} \rightarrow Y$ to the $n$-cells of $X$. What restrictions are there on the degrees of the map on the $n$-cells?

Let $\alpha \in \pi_{n-1}\left(X^{(n-1)}\right)$ be the attaching map of an $n$-cell, and consider the pair of exact sequences

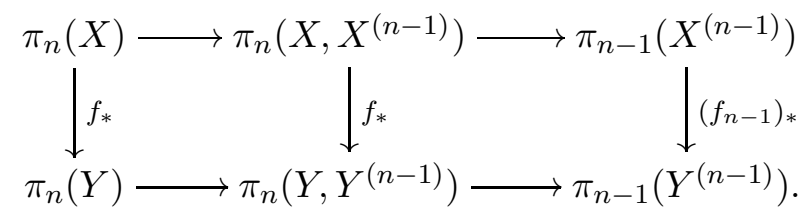

Since $\pi_{n}\left(Y, Y^{(n-1)}\right) \cong H_{n}(Y)$, the middle arrow controls the degrees we are interested in; since the diagram commutes, they are determined up to the Hurewicz image of $\pi_{n}(Y)$. For example, if $X$ and $Y$ each have a single $n$-cell which kills a rationally nontrivial element of $\pi_{n-1}$, then the Hurewicz image is trivial and the degree on that cell is determined by the homotopy type of $f_{n-1}$. This will be used in the construction in Section 3.

This observation was used in CDMW18 to show that certain homotopically trivial maps are hard to nullhomotope. For example, let $Z_{4}$ be the space obtained from $S^{2} \vee S^{2}$ by gluing on two 5-cells killing $\pi_{4}\left(S^{2} \vee S^{2}\right) \otimes \mathbb{Q}$. While $\pi_{4}\left(Z_{4}\right)$ is trivial, an $L$-Lipschitz map $S^{4} \rightarrow Z_{4}$ may only be nullhomotopic via an $\Omega\left(L^{6 / 5}\right)$-Lipschitz homotopy. The argument showing this is very similar in flavor to the arguments in this paper.

2.3. Smoothly formal spaces. We say that a piecewise Riemannian space $Y$ is smoothly formal if there is a splitting algebra homomorphism $H^{*}(Y ; \mathbb{Q}) \rightarrow \Omega^{*}(Y)$. Note that a smoothly formal space is in particular formal in the sense of Sullivan. The only examples of smoothly formal spaces we are aware of are wedges of Riemannian symmetric spaces.

The quantitative homotopy theory of such spaces is particularly nice. In particular, we have the following theorem, contrasting with the observation about the space $Z_{4}$ in the previous subsection.

Theorem 2.3 (see $\S 5.3$ of [Man18]). If $X$ and $Y$ are finite complexes and $Y$ has the rational homotopy type of a simply connected smoothly formal space, then every nullhomotopic L-Lipschitz map $X \rightarrow Y$ has an $O(L \exp (\kappa(X, Y) \sqrt{\log L}))$-Lipschitz nullhomotopy. In particular, for $X=S^{n}$ (or any other co-H-space), any two homotopic L-Lipschitz maps $X \rightarrow Y$ are homotopic via a $O(L \exp (\kappa(X, Y) \sqrt{\log L}))$-Lipschitz homotopy.

This known bound is $o\left(L^{1+\varepsilon}\right)$ for every $\varepsilon>0$. In fact, I believe that a linear bound can be attained. This conjecture is recorded here as its truth would significantly strengthen the results of this paper.

Conjecture 2.4. If $X$ and $Y$ are finite complexes and $Y$ has the rational homotopy type of a simply connected smoothly formal space, then every nullhomotopic L-Lipschitz map $X \rightarrow Y$ has an $O(L)$-Lipschitz nullhomotopy.

2.4. Wedges of spheres. An important class of smoothly formal spaces is wedges of spheres. Here we describe the homotopy theory of the space $\bigvee_{i} S^{n_{i}}$.

Let $X$ be any space. Given maps $f: S^{i} \rightarrow X$ and $g: S^{j} \rightarrow X$, their Whitehead product $[f, g]: S^{i+j-1} \rightarrow X$ is the composition

$$
S^{i+j-1} \stackrel{\text { attaching map of the }(i+j) \text {-cell of } S^{i} \times S^{j}}{\longrightarrow} S^{i} \vee S^{j} \stackrel{f \vee g}{\longrightarrow} X .
$$


Note that while we defined the Whitehead product of two maps, the Whitehead product is also well-defined on the level of homotopy groups. (We will use the same notation for the mapand homotopy-level products.) In fact, up to homotopy, it is bilinear, graded commutative, and satisfies the graded Jacobi identity

$$
(-1)^{i j}[f,[g, h]]+(-1)^{j k}[g,[h, f]]+(-1)^{k i}[h,[f, g]]=0,
$$

for $f: S^{i} \rightarrow X, g: S^{j} \rightarrow X$, and $h: S^{k} \rightarrow X$. In other words, the Whitehead product defines a graded Lie bracket on the graded module $\pi_{*+1}(X)$ (i.e. elements of $\pi_{2}(X)$ have degree 1 , etc.)

For $X=\bigvee_{i} S^{n_{i}}$, the rationalization $\pi_{*+1}(X) \otimes \mathbb{Q}$ is in fact a free graded Lie algebra over $\mathbb{Q}$ generated by an $\left(n_{i}-1\right)$-dimensional generator for each $n_{i}$.

Given an ordering on the generators of a free Lie algebra over a field of characteristic zero, one obtains a Hall set of monomials which form a basis for the Lie algebra as a vector space. It is not hard to see that this Hall set, together with $[a, a]$ for every element $a$ of odd degree in the Hall set, still forms a basis for the free graded Lie algebra; cf. [Hil55], where this is shown for the homotopy algebra of a wedge of spheres of the same dimension. We follow Hilton's formulation of the Hall set, which differs slightly from some more modern treatments.

\section{THE ZOO}

In this section we give a large number of simple examples of spaces $X$ and $Y$ for which the growth of $[X, Y]$ can be determined enough to rule out polynomial behavior. Each is given by a few cells with attaching maps given by iterated Whitehead products.

Example 3.1. Let $X=\left(S^{3} \vee S^{4}\right) \cup_{\left[\mathrm{id}_{S^{3}},\left[\operatorname{id}_{S^{3}}, \operatorname{id}_{S^{4}}\right]\right]} D^{9}$, and let $Y$ be the space obtained from $X$ by adding 10-cells to kill $\pi_{9}(X)$. Then the growth of $[X, Y]$ is $O\left(L^{13 / 2}\right)$ and $\omega\left(L^{13 / 2-\varepsilon}\right)$ for every $\varepsilon>0$.

Note that purely obstruction-theoretic concerns would suggest that there should be $\Theta\left(L^{7}\right)$ homotopy classes of $L$-Lipschitz maps in this case as well as that of Example 3.5. Indeed, in both cases the growth turns out to be slower.

If we assume Conjecture 2.4, the $O\left(L^{13 / 2}\right)$ bound is sharp by the same argument using the conjecture instead of Theorem 2.3 ,

Proof. By the discussion in 32.1 , we can assume that maps $X \rightarrow Y$ are cellular. By obstruction theory, since $H^{k}\left(X ; \pi_{k}(Y)\right)$ is only nonzero in degrees 3 and 4 , the homotopy class of such a map is determined by the degrees of the restriction-projections $\alpha: S^{3} \rightarrow S^{3}$ and $\beta: S^{4} \rightarrow S^{4}$ and a $\mathbb{Z} / 2 \mathbb{Z}$ invariant $\tau$ coming from $\pi_{4}\left(S^{3}\right)$. In turn, the two degrees determine the degree on the 9-cell of $X$ : the boundary of this cell is mapped to $S^{3} \vee S^{4} \subset Y$ via $[\alpha,[\alpha, \beta \vee \tau]]$, and the bilinearity of the Whitehead product means that any nullhomotopy of this boundary map in $Y$ must have degree $(\operatorname{deg} \alpha)^{2} \operatorname{deg} \beta$.

Assume to simplify notation that the metric on $Y$ extends that on $X$. A cellular map with Lipschitz constant $\leq L$ must then satisfy

$$
\left\{\begin{aligned}
|\operatorname{deg} \alpha| & \leq L^{3} \\
|\operatorname{deg} \beta| & \leq L^{4} \\
\left|(\operatorname{deg} \alpha)^{2} \operatorname{deg} \beta\right| & \leq L^{9} .
\end{aligned}\right.
$$

An integral shows that the area of the region in $\mathbb{R}^{2}$ satisfying these conditions is $O\left(L^{13 / 2}\right)$; the number of lattice points is similar. This demonstrates the upper bound. 
For the lower bound, we need to actually exhibit $\Omega\left(L^{13 / 2-\varepsilon}\right)$ different maps whose Lipschitz constant is $O(L)$, for every $\varepsilon>0$. So fix $\varepsilon$, and consider any $a, b \in \mathbb{Z}$ satisfying

$$
\left\{\begin{aligned}
|a| & \leq L^{3-\varepsilon} \\
|b| & \leq L^{4-\varepsilon} \\
\left|a^{2} b\right| & \leq L^{9-2 \varepsilon} .
\end{aligned}\right.
$$

We will construct a cellular $O(L)$-Lipschitz map $f_{a, b}: X \rightarrow Y$ whose degrees on the 3- and 4-cell are $a$ and $b$, respectively. Write $u_{k, d}: S^{k} \rightarrow S^{k}$ for a maximally efficient, hence $O\left(d^{1 / k}\right)$ Lipschitz map of degree $d$. We start by setting $f_{a, b}$ to be $u_{3, a}$ on the 3 -cell and $u_{4, b}$ on the 4-cell. All that is left is to extend to the 9-cell.

As a shorthand, given a map $f: S^{k} \rightarrow S^{k}$, we write $d \cdot f$ to mean $f \circ u_{k, d}$ (a particular efficient representative of the homotopy class $d[f]$.) Then let $g_{1}: S^{8} \rightarrow S^{3} \vee S^{4}$ be given by $\left[u_{3, a},\left[u_{3, a}, u_{4, b}\right]\right]$ and $g_{2}: S^{8} \rightarrow S^{3} \vee S^{4}$ be given by

$$
S^{8} \stackrel{\text { pinch the equator }}{\longrightarrow} S^{8} \vee S^{8} \stackrel{\left[s \cdot \operatorname{id}_{S^{3}}, t \cdot\left[\mathrm{id}_{S^{3}}, \mathrm{id}_{S^{4}}\right]\right] \vee e \cdot\left[\operatorname{id}_{S^{3}},\left[\operatorname{id}_{S^{3}}, \mathrm{id}_{S^{4}}\right]\right]}{\longrightarrow} S^{3} \vee S^{4},
$$

where $s=\left\lfloor L^{3-\varepsilon}\right\rfloor, t=\left\lfloor L^{-(3-\varepsilon)} a^{2} b\right\rfloor$, and $e=a^{2} b-s t$ is an $O\left(L^{6}\right)$ correction term that makes $g_{1}$ and $g_{2}$ homotopic. Note that $g_{1}$ and $g_{2}$ are both $O\left(L^{1-\varepsilon / 4}\right)$-Lipschitz. Then by Theorem 2.3, there is an $O(L)$-Lipschitz homotopy between them in $S^{3} \vee S^{4}$.

Moreover, $g_{2}$ extends to an $O\left(L^{1-\varepsilon / 4}\right)$-Lipschitz map $D^{9} \rightarrow Y$. We demonstrate this by giving separate nullhomotopies of

$$
\left[s \cdot \operatorname{id}_{S^{3}}, t \cdot\left[\operatorname{id}_{S^{3}}, \operatorname{id}_{S^{4}}\right]\right] \text { and } e \cdot\left[\operatorname{id}_{S^{3}},\left[\operatorname{id}_{S^{3}}, \operatorname{id}_{S^{4}}\right]\right] .
$$

The second of these maps has an $O\left(L^{3 / 4}\right)$-Lipschitz nullhomotopy given by composing the attaching map $D^{9} \rightarrow Y$ of the 9-cell with the cone $C u_{8, e}: D^{9} \rightarrow D^{9}$ of $u_{8, e}$. The first map has an $O\left(L^{1-\varepsilon / 4}\right)$-Lipschitz nullhomotopy factoring through a sequence of maps

$$
D^{9} \stackrel{\text { top cell }}{\longrightarrow} S^{3} \times S^{6} \stackrel{u_{3, s} \times u_{6, t}}{\longrightarrow} S^{3} \times S^{6} \rightarrow X \subset Y
$$

here the last arrow sends $* \times S^{6}$ to $S^{3} \vee S^{4}$ via the Whitehead product.

Finally, we extend $f_{a, b}$ to the 9-cell of $X$ by concatenating the homotopy from $g_{1}$ to $g_{2}$ and the nullhomotopy of $g_{2}$.

We now give the more general version. The proof is abbreviated since it is very similar to the one above once $X$ and $Y$ are constructed.

Theorem 3.4. For any rational number $r>4$, there are spaces $X$ and $Y$ such that the growth of $[X, Y]$ is $O\left(L^{r}\right)$ and $\omega\left(L^{r-\varepsilon}\right)$ for any $\varepsilon>0$.

Proof. We fix natural numbers $\ell<m$ and $1 \leq p<q$ such that

$$
r=\ell+m+\frac{2-p-q}{q} \text {. }
$$

Note that every rational number in $(\ell+m-2, \ell+m-1]$ can be written this way for some $p$ and $q$. In addition we set $\ell$ and $m$ so that $\ell \geq 2$ and $m \geq 4$ and $m-\ell$ is 1 or 2. Fix

$$
n=p(\ell-1)+q(m-1)+2 .
$$

Let $\zeta \in \pi_{n-1}\left(S^{\ell} \vee S^{m}\right)$ be given by the iterated Whitehead product

$$
\left.\left.\left.\left.\zeta=[\underbrace{\operatorname{id}_{S^{\ell}},\left[\cdots \left[\operatorname{id}_{S^{\ell}}\right.\right.}_{p-1 \text { times }},[\underbrace{\operatorname{id}_{S^{m}},\left[\cdots \left[\operatorname{id}_{S^{m}}\right.\right.}_{q \text { times }}, \operatorname{id}_{S^{\ell}}] \cdots]\right]\right] \cdots\right]\right] .
$$


This is an element of infinite order since, for the ordering $\operatorname{id}_{S^{m}}<\mathrm{id}_{S^{\ell}}$, it is a Hall element of the free Lie algebra. We let $X=\left(S^{\ell} \vee S^{m}\right) \cup_{\zeta} D^{n}$ and $Y$, like before, is obtained from $X$ by adding $(n+1)$-cells to kill $\pi_{n}(X)$. Then a cellular map $X \rightarrow Y$ with Lipschitz constant $L$ is determined (up to an element of the finite group $\pi_{m}\left(S^{\ell}\right)$ ) by the degrees $a$ and $b$ of the restrictions to $S^{\ell} \rightarrow S^{\ell}$ and $S^{m} \rightarrow S^{m}$. These degrees must satisfy

$$
\left\{\begin{aligned}
|a| & \leq L^{\ell} \\
|b| & \leq L^{m} \\
\left|a^{p} b^{q}\right| & \leq L^{n}
\end{aligned}\right.
$$

The volume of this region in $\mathbb{R}^{2}$ is $\Theta\left(L^{r}\right)$.

As before, for any $\varepsilon>0$, we can actually realize $\Omega\left(L^{r-\varepsilon}\right)$ elements which satisfy corresponding slightly stronger bounds. To do this, we take a map $S^{\ell} \vee S^{m} \rightarrow Y$ with degrees $a, b$ satisfying such bounds and extend it to the $n$-cell of $X$ via a concatenation of two homotopies: one from the induced map on the boundary of this cell to a Whitehead product

$$
\left.\left.\left.\left.\left[\left\lfloor L^{\ell-\varepsilon}\right\rfloor \cdot \operatorname{id}_{S^{\ell}},\left\lfloor L^{-(\ell-\varepsilon)} a^{p} b^{q}\right\rfloor \cdot[\underbrace{\operatorname{id}_{S^{\ell}},\left[\cdots \left[\operatorname{id}_{S^{\ell}}\right.\right.}_{p-2 \text { times }},[\underbrace{\operatorname{id}_{S^{m}},\left[\cdots \left[\operatorname{id}_{S^{m}}\right.\right.}_{q \text { times }}, \operatorname{id}_{S^{\ell}}] \cdots]\right]\right] \cdots\right]\right]\right]
$$

together with an $O\left(L^{n-\ell}\right)$ correction term, followed by a nullhomotopy of that map which factors through $S^{\ell} \times S^{m}$.

Example 3.5. Let $X=\left(S^{3} \vee S^{4}\right) \cup_{\left[\left[\mathrm{id}_{S^{3}}, \mathrm{id}_{S^{4}}\right],\left[\operatorname{id}_{S^{3}}, \operatorname{id}_{S^{4}}\right]\right]} D^{12}$, and let $Y$ be the space obtained from $X$ by adding 13-cells killing $\pi_{12}(X)$. Then the growth of $[X, Y]$ is $\Theta\left(L^{6} \log L\right)$.

Proof. This example is in many ways similar to the previous ones. Once again, an $L$-Lipschitz map $X \rightarrow Y$ is determined up to a finite kernel by two degrees $a$ and $b$ which satisfy

$$
\left\{\begin{aligned}
|a| & \leq L^{3} \\
|b| & \leq L^{4} \\
\left|a^{2} b^{2}\right| & \leq L^{12}
\end{aligned}\right.
$$

integrating gives $\Theta\left(L^{6} \log L\right)$ such pairs.

However, in this case we can explicitly construct an $O(L)$-Lipschitz map for every such pair of degrees $(a, b)$, using a refinement of the construction in Theorem 3.4. On the 3- and 4-cells, we fix explicit $O(L)$-Lipschitz maps $w_{a}: S^{3} \rightarrow S^{3}$ and $w_{b}: S^{4} \rightarrow S^{4}$ of the correct degree. Then on the 12-cell, the map is a concatenation of two homotopies which together form a nullhomotopy of the Whitehead square $\left[\left[w_{a}, w_{b}\right],\left[w_{a}, w_{b}\right]\right]$.

The first homotopy is a fiberwise Whitehead square $\left(h_{1}\right)_{t}=\left[g_{t}, g_{t}\right]$, where $g: S^{6} \times[0,1] \rightarrow$ $S^{3} \vee S^{4}$ is an $O(L)$-Lipschitz homotopy (constructed explicitly later) which takes

$$
\left[w_{a}, w_{b}\right] \simeq_{g_{t}} a b \cdot\left[\operatorname{id}_{S^{3}}, \operatorname{id}_{S^{4}}\right] .
$$

Note that the map at time 1 is $O(L)$-Lipschitz because $a b \leq L^{6}$; in the analogous step in Example 3.1, where $a b$ may be greater, we needed to exploit the bilinearity of both Whitehead products simultaneously, making an explicit construction harder to obtain.

We concatenate this with a nullhomotopy of

$$
\left[a b \cdot\left[\operatorname{id}_{S^{3}}, \operatorname{id}_{S^{4}}\right], a b \cdot\left[\operatorname{id}_{S^{3}}, \operatorname{id}_{S^{4}}\right]\right]
$$

which proceeds via the $(O(L)$-Lipschitz) composition

$$
D^{12} \stackrel{\text { attaching map }}{\longrightarrow} S^{6} \times S^{6} \stackrel{u_{6, a b} \times u_{6, a b}}{\longrightarrow} S^{6} \times S^{6} \rightarrow X \subset Y,
$$




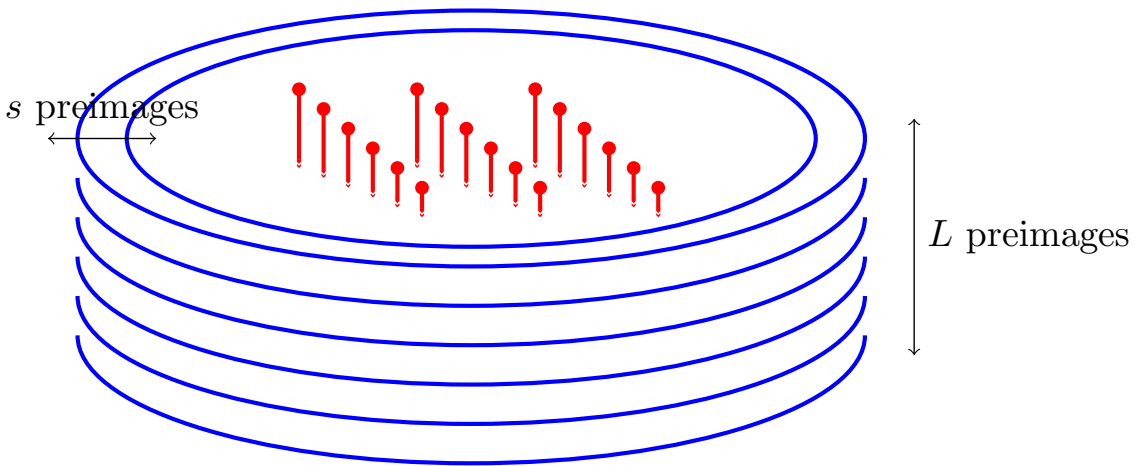

Figure 1. The chosen cylinder $S^{i-1} \times D^{j}$ when $i=j=2$; in this case the pancakes are horizontal slices, arranged in a stack. The blue and red lines are preimages of the north pole of $S^{i}$ and $S^{j}$, respectively; the red ones wrap all the way around, outside this chosen subdomain.

where the last arrow takes each of the 6-cells to $S^{3} \vee S^{4}$ via the Whitehead product.

The key part of the proof is specifying the maps $w_{a}, w_{b}$, and $g$ more precisely in order to ensure that everything is $O(L)$-Lipschitz. If we knew Conjecture 2.4, we would obtain the existence of such a homotopy automatically, but this case is simple enough that we can do it by hand. Roughly speaking, we keep track of the preimages of the north pole of $S^{3}$ (a 3 -manifold) and the north pole of $S^{4}$ (a 2-manifold). Each preimage starts out as a grid of spheres, and these grids are linked together; gradually, we tease the links apart, so that at the end the preimages consist of $\leq L^{6}$ pairs of linked $S^{2}$ and $S^{3}$. So that this actually extends to an $O(L)$-Lipschitz homotopy, we make sure that the bordisms have non-overlapping embedded normal neighborhoods of radius $\Omega(1 / L)$. We now give the construction in more detail; the bordisms themselves are laid out in Figure 2 ,

We first specify $w_{a}$. We tile the bottom of a unit 3-cube with $a$ identical open balls of diameter $1 / L, L$ to a side. We get $\left\lceil a / L^{2}\right\rceil$ layers of such balls, with the last layer incomplete. Fix an embedding of this cube in $S^{3}$; we let the map $w_{a}: S^{3} \rightarrow S^{3}$ send every point outside the balls inside the cube to the basepoint $*$, and each of the balls homeomorphically to $S^{3} \backslash *$. We construct $w_{b}$ analogously. Note that

$$
\left\lceil\frac{a}{L^{2}}\right\rceil \cdot\left\lceil\frac{b}{L^{3}}\right\rceil \leq 2 L
$$

We now describe the homotopy $g$; this is most easily done replacing the dimensions 3 and 4 by some general $i, j \geq 2$, and 6 by $i+j-1$. We give a pictorial step-by-step construction of $g$ which may be taken literally when $i, j=2$ but is easily seen to work for other $i, j$.

The domain $S^{i+j-1}$ of $\left[w_{a}, w_{b}\right]$ can be split into two solid tori: the preimage $D^{i} \times S^{j-1}$ of $S^{i}$, on which the map is $w_{a}$ after projection onto the first coordinate, and the preimage $S^{i-1} \times D^{j}$ of $S^{j}$ on which the map is $w_{b}$ after projection onto the second coordinate.

Within the first torus, we have a $[0,1]^{i} \times S^{j-1}$, the subspace on which $w_{a}$ is nontrivial. Let's say that the last coordinate represents "depth"; then we complete this $[0,1]^{i-1} \times\left([0,1] \times S^{j-1}\right)$ to a cylinder $[0,1]^{i-1} \times D^{j}$ where the map on each filling $* \times D^{j}$ restricts to $w_{b}$. We then slice this cylinder into $L^{i-1}$ thickened $j$-disks which we call "pancakes"; the case $i=j=2$ is shown in Figure 1. Let $s=\left\lceil a / L^{i-1}\right\rceil$ and $t=\left\lceil b / L^{j-1}\right\rceil$ be the depths to which we filled the 


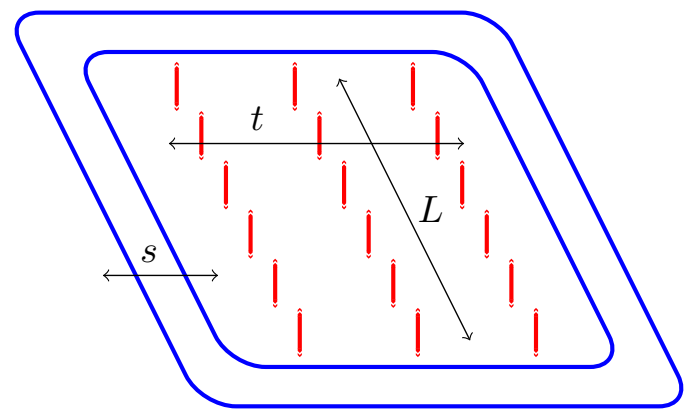

(A) The initial preimages of the north poles of $S^{i}$ (blue) and $S^{j}$ (red) inside a single pancake.

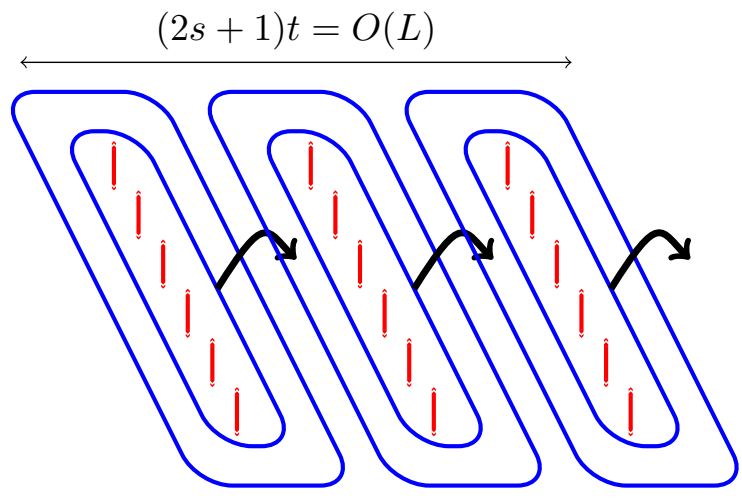

(c) ... separating the preimages of $S^{j}$ into layers, each encircled by preimages of $S^{i}$. Then we take the skinny rings one by one...

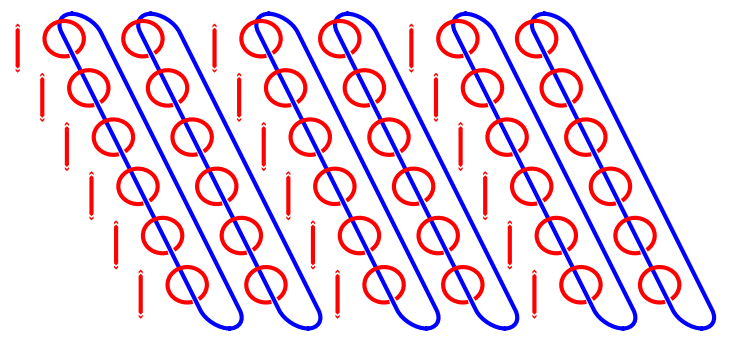

(E) Finally, we separate the $L^{j-1}$-part links into Hopf links, and simultaneously...

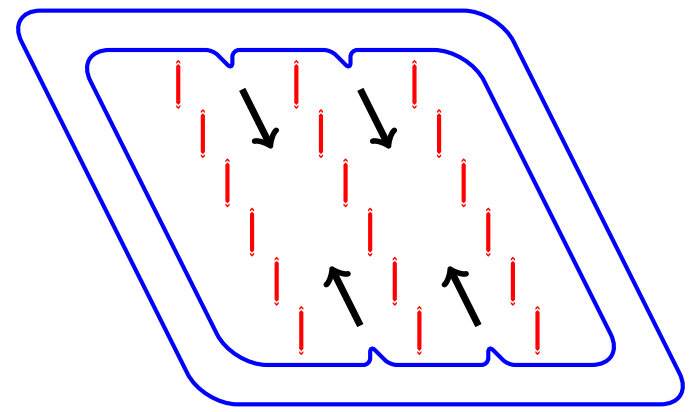

(B) The first step is to push the preimages of $S^{i}$ inwards...

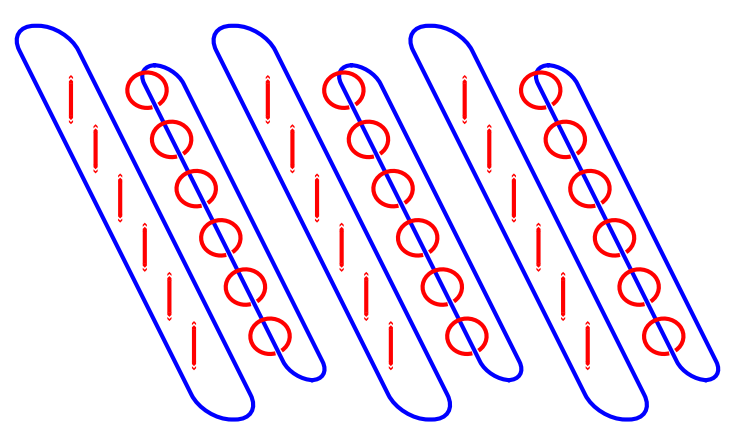

(D) ... and bud them off, creating a new layer for each.

\section{Q) Q Q Q Q Q Q Q Q) Q Q Q Q Q Q Q) Q Q Q Q Q Q Q) Q Q Q Q Q Q Q Q) Q Q Q Q Q Q Q Q Q Q}

(F) ... kill the big leftover preimages of $S^{j}$ which are no longer linked with anything. In the end, we have a grid of Hopf links, as desired.

FiguRE 2. The stages of the homotopy $g$, as seen on the neighborhood of a $j$-dimensional cross-section. The preimage of the north poles of $S^{i}$ and $S^{j}$ are shown in blue and red, respectively, and the homotopy corresponds to a simultaneous bordism of these two manifolds. The vertical direction stands in for a $(j-1)$-dimensional subspace.

two cubes to build $w_{a}$ and $w_{b}$; then each pancake contains about $s$ strands of the preimage of $S^{i}$.

We now give the construction of the previously mentioned bordism; we do this the same way within each pancake, as shown in Figure 2. 


\section{SyMmetric SPACES}

As shown in [MW18], the growth of $[X, Y]$ still need not be polynomial even when $Y$ is the smoothly formal space $S^{4}$. However, in this case the assumption (S2) given in the introduction can be formalized, and holds, or nearly so. We will show this here for symmetric spaces. The argument easily extends to wedges of symmetric spaces, but to give the correct statement there would require some extra definitions which would take us too far afield.

In this section we take the naive, skeleton-by-skeleton approach to constructing efficient maps. For example, given a simplicial complex $X$ and an odd $n \geq 3$, maps $X \rightarrow S^{n}$ are determined up to a finite indeterminacy by an obstruction class in $H^{n}(X)$. Given a simplicial cochain $w \in C^{n}(X)$ representing this homology class, we can construct a map as follows:

- Send the $(n-1)$-skeleton to the basepoint of the sphere.

- Send each $n$-simplex to an optimally efficient map whose degree is dictated by the given cochain.

- Extend to higher skeleta. For each $k$-simplex, we have a finite number of choices up to homotopy in non-canonical bijection with $\pi_{k}\left(S^{n}\right)$.

The Lipschitz constant of the map on $X^{(n)}$ generated by the first two steps is $C(n)\left(\|w\|_{\infty}\right)^{1 / n}$. However, to do the later steps in a quantitative way, one also needs bounds on the size of the smallest map filling in a given map on the boundary of a $k$-simplex; in other words, estimates on the size of a nullhomotopy. Luckily, Theorem B of CDMW18 shows that one can construct linear nullhomotopies in this situation, and therefore the Lipschitz constant of the resulting map is linear in $\left(\|w\|_{\infty}\right)^{1 / n}$. Moreover, by Lemma 2.1, it is sharp up to a constant: every $L$-Lipschitz map has a first obstruction $w$ with $\|w\|_{\infty} \leq C L^{n}$.

Things get more complicated if we look at maps $X \rightarrow S^{n}$ for $n$ even. Here there is a second rational obstruction in dimension $2 n-1$; more importantly, we no longer have a linear bound on the sizes of nullhomotopies, unless we assume Conjecture 2.4. Nevertheless, we can follow the same procedure as above up to the $(2 n-2)$-skeleton, and then perform the following steps:

- Fill in the $(2 n-1)$-simplices in the most efficient possible way.

- Now there is an obstruction in $H^{2 n-1}(X)$ to extending to our desired homotopy class. Given a cochain $z$ representing this obstruction, we glue in maps $S^{2 n-1} \rightarrow S^{n}$ on each simplex as dictated by $z$. These maps can have Lipschitz constant $\leq C(n)\left(\|z\|_{\infty}\right)^{1 / 2 n}$, as per Gro78.

- Finally we extend to higher skeleta as before. However, this time our extensions are only guaranteed by Theorem 2.3 to be $O\left(L^{1+\varepsilon}\right)$-Lipschitz for any given $\varepsilon>0$, where $L$ is the Lipschitz constant of the map on the previous skeleton.

Thus as we go up, we accumulate nonlinearity. However, in the end, we can still ensure that the Lipschitz constant is at most

$$
C(n, \operatorname{dim} X, \varepsilon)\left(\|w\|_{\infty}^{1 / n}+\|z\|_{\infty}^{1 / 2 n}\right)^{1+\varepsilon}
$$

for any $\varepsilon>0$ we choose. The converse is a bit harder to state, since $z$ is a relative obstruction. Given an $L$-Lipschitz map, we again have $\|w\|_{\infty} \leq C(n) L^{n}$; moreover, given two maps which coincide on $X^{(2 n-2)}$, the obstruction $z^{\prime}$ to homotoping them in dimension $2 n-1$, which is well-defined, satisfies $\left\|z^{\prime}\right\|_{\infty} \leq C(n) L^{2 n}$. Thus the total number of homotopy classes of $L$-Lipschitz maps is

$$
O\left(L^{n \text { rk } H^{n}(X)+2 n \operatorname{rk} H^{2 n-1}(X)}\right),
$$


although it could be much smaller since different obstructions may specify the same homotopy class. The main theorem of this section uses the results of [Man18] to generalize these observations to spaces with the rational homotopy type of symmetric spaces.

Now, more generally, let $Y$ be a finite CW complex whose 1-skeleton is a point. Then a homotopy class of maps $X \rightarrow Y$ is specified (in an obviously non-unique way) by a sequence of obstructions in $C^{k}\left(X ; \pi_{k}(Y)\right), k=2, \ldots, \operatorname{dim} X$. More precisely, the $k$ th such obstruction sends each $k$-simplex $p$ to an element of the torsor for $\pi_{k}(Y)$ consisting of nullhomotopies of the map on $\partial p$. Thus it is only well-defined once the map is fixed on lower skeleta. Nevertheless, as in the case of even-dimensional spheres, we can still compare the sizes of obstructions realizable with Lipschitz constant $L$.

Theorem 4.1. Let $X$ and $Y$ be finite complexes such that $Y$ has the rational homotopy type of a symmetric space. Then there is a way to make sense of the "size" of a sequence of obstructions so that, assuming Conjecture 2.4, the minimal Lipschitz constant of a representative of $\alpha \in[X, Y]$ is equal up to a multiplicative constant to the minimal size of a sequence of obstructions describing it. Without that assumption, they are related in at worst a slightly superlinear way.

Specifically:

- Denote the rational Hurewicz map by $h_{k}: \pi_{k}(Y) \otimes \mathbb{Q} \rightarrow H_{k}(Y ; \mathbb{Q})$.

- For every $k$, fix a splitting

$$
\pi_{k}(Y) \otimes \mathbb{Q}=\operatorname{ker} h_{k} \oplus N_{k}
$$

with projections $p_{1}, p_{2}$ sending elements to $\operatorname{ker} h_{k}$ and $N_{k}$ respectively, and a norm $|\cdot|$ on $\left.\pi_{k}(Y) \otimes \mathbb{Q}\right)$.

Then there are a constant $c>0$ and a function $g: \mathbb{R}^{+} \rightarrow \mathbb{R}^{+}$depending on these norms, and a choice of zero for every torsor described above, such that:

(i) Every cL-Lipschitz map lies in a homotopy class which can be described by obstructions $\beta_{k} \in C^{k}\left(X ; \pi_{k}(Y)\right)$ such that

$$
\sup _{k \text {-cells } \sigma \text { of } X}\left|p_{1}\left(\beta_{k}(\sigma)\right)\right| \leq L^{k+1} \quad \text { and } \quad \sup _{k \text {-cells } \sigma \text { of } X}\left|p_{2}\left(\beta_{k}(\sigma)\right)\right| \leq L^{k} .
$$

(ii) Conversely, every homotopy class which can be described by such obstructions (even just rationally) has a $g(L)$-Lipschitz representative.

Assuming Conjecture 2.4, this $g$ is linear. Otherwise, we merely know that $g(L)=O\left(L^{1+\varepsilon}\right)$ for every $\varepsilon>0$.

Proof. The proof uses the following corollary of [Man18, Thm. 5.3]:

Theorem 4.2. Define $S_{L, c} \subset \pi_{n}(Y)$ to be

$$
\left\{\alpha \in \pi_{n}(Y):\left|p_{1}(\alpha)\right| \leq c L^{k+1} \text { and }\left|p_{2}(\alpha)\right| \leq c L^{k}\right\} \text {. }
$$

Then there are constants $C>c>0$ such that the set of elements of $\pi_{n}(Y)$ which have $L$-Lipschitz representatives lies between $S_{L, c}$ and $S_{L, C}$.

To show part (i), we take two $L$-Lipschitz maps (which we can assume to be cellular) and let $k$ be the first dimension in which they differ. Then the obstruction to homotoping one to the other is an element of $C^{k}\left(X ; \pi_{k}(Y)\right)$ where every cell $\sigma$ is sent to a $C_{\sigma} L$-Lipschitz map $S^{k} \rightarrow Y$. Then Theorem 4.2 gives the right kind of bound on the size of this obstruction.

Now we show part (ii). Given an obstruction-theoretic description of a homotopy class $\alpha \in[X, Y]$, we build a map $f: X \rightarrow Y$ by skeleta. First send $X^{(1)}$ to the basepoint. Now 
suppose we have constructed $f_{k-1}: X^{(k-1)} \rightarrow Y$ with Lipschitz constant $M$. We extend to $X^{(k)}$ by first using the bound of Theorem 2.3 (or that of Conjecture 2.4) to fill in the $k$ simplices as efficiently as possible, then in each $k$-simplex $p$ glue in an efficient representative of the element $\alpha_{p} \in \pi_{k}(X)$ corresponding to the resulting obstruction. The Lipschitz constant of the resulting map is

$$
C_{k} \max \left\{\gamma_{k}(M), \sup \left\{\operatorname{Lip} \alpha_{p}: k \text {-simplices } p \text { of } X\right\}\right\},
$$

where $\gamma_{k}$ is our bound on sizes of homotopies.

If all the $\gamma_{k}$ are linear, we get a linear bound. Otherwise, we accumulate a Lipschitz constant

but this is still $O\left(L^{1+\varepsilon}\right)$ for any $\varepsilon>0$.

$$
\gamma_{\operatorname{dim} X}\left(\cdots\left(\gamma_{3}\left(\gamma_{2}(L)\right)\right) \cdots\right)
$$

Now, maps in the same rational homotopy class may not be specified by the same rational obstructions. However, they have the same optimal Lipschitz constant by Theorem 5.1 of Man18].

Acknowledgments. The author was partially supported by NSF RTG grant DMS-1547357. He would like to thank the anonymous referee for helpful suggestions regarding the exposition.

\section{REFERENCES}

[CDMW18] Gregory R Chambers, Dominic Dotterrer, Fedor Manin, and Shmuel Weinberger, Quantitative null-cobordism, J. Amer. Math. Soc. 31 (2018), no. 4, 1165-1203.

[Gro78] Mikhail Gromov, Homotopical effects of dilatation, Journal of Differential Geometry 13 (1978), no. 3, 303-310.

[Gro81] _ Groups of polynomial growth and expanding maps, Publications Mathématiques de l'IHÉS (1981), no. 53, 53-73. MR 623534

[Gro98] ㄴ. Metric structures for Riemannian and non-Riemannian spaces, vol. 152, Birkhäuser Boston, 1998.

[Gro99] _ Quantitative homotopy theory, Invited Talks on the Occasion of the 250th Anniversary of Princeton University (H. Rossi, ed.), Prospects in Mathematics, 1999, pp. 45-49.

[Hil55] P.J. Hilton, On the homotopy groups of the union of spheres, Journal of the London Mathematical Society 30 (1955), no. 2, 154-172.

[Man18] Fedor Manin, Plato's cave and differential forms, arXiv preprint arXiv:1801.00335 (2018).

[MW18] Fedor Manin and Shmuel Weinberger, Integral and rational mapping classes, arXiv preprint arXiv:1802.05784 (2018). 